Rabaska

Revue d'ethnologie de l'Amérique française

\title{
« Nos traditions à l'Université » : un rappel
}

\section{Jean-Pierre Pichette}

Volume 13, 2015

Présence de Marius Barbeau : l'invention du terrain en Amérique française. Autour d'un legs centenaire (1914-2014)

URI : https://id.erudit.org/iderudit/1033767ar

DOI : https://doi.org/10.7202/1033767ar

Aller au sommaire du numéro

Éditeur(s)

Société québécoise d'ethnologie

ISSN

1703-7433 (imprimé)

1916-7350 (numérique)

Découvrir la revue

Citer cet article

Pichette, J.-P. (2015). « Nos traditions à l’Université » : un rappel. Rabaska, 13, 263-266. https://doi.org/10.7202/1033767ar

Ce document est protégé par la loi sur le droit d'auteur. L'utilisation des services d'Érudit (y compris la reproduction) est assujettie à sa politique d'utilisation que vous pouvez consulter en ligne.

https://apropos.erudit.org/fr/usagers/politique-dutilisation/ 


\section{Nos traditions à l'Université}

\section{« Nos traditions à l'Université » : un rappel ${ }^{1}$ \\ JeAn-Pierre Pichette \\ Société québécoise d'ethnologie Professeur associé, Université Sainte-Anne}

\section{Contexte}

Un soir de l'été 1952, dans le Quartier latin, près des remparts, un anthropologue réputé, un prêtre et un jeune professeur enregistrent, chacun à sa façon, le conte d'un pêcheur acadien de passage à Québec. Ce dernier n'entrevoit guère la commotion que produira sur son savant auditoire le petit fabliau qu'il lui débite.

L'événement met ainsi en scène, d'une part, trois personnages dont l'amitié, qui remonte à trois lustres, et la commune passion pour les traditions orales ont engendré une dizaine d'années plus tôt la création d'une chaire de folklore à l'Université Laval. Ces pères trinitaires du folklore canadien-français sont Marius Barbeau (1883-1969), un vert sexagénaire, polygraphe, dont le glas seul sonnera l'heure de la retraite; Félix-Antoine Savard (1896-1982), prêtre écrivain au cœur de la cinquantaine, dont le Menaud, maître draveur est déjà une œuvre de renom ; et Luc Lacourcière (1910-1989), folkloriste au début de la quarantaine, qui, depuis douze ans, fréquente autant le peuple que les livres.

Devant eux, d'autre part, un jeune Acadien de 19 ans, Onias Ferron, de Saint-Raphaël-sur-Mer, au Nouveau-Brunswick, que Lacourcière et Savard connaissent depuis 1951 et de qui ils ont recueilli, dans son pays en 1952, cinq chansons, six contes et un reel à bouche. Ce soir-là, leur attention sera drainée vers un seul conte, minuscule, d'à peine plus de deux minutes, « La

1. Marius Barbeau, « Nos traditions à l'Université », Journal of American Folklore, vol. 67, $\mathrm{n}^{\circ}$ 264, avril-juin 1954, p. 199-211. 
Sereine de mer et les trois haches ${ }^{2} »$, que le narrateur tient depuis peu de son père, Alexandre Ferron ; celui-ci le tenait peut-être de son propre père, Antoine (Pitou) Ferron, qui avait « une réputation de conteur remarquable». Les auditeurs découvrent alors, fascinés, la version orale d'un fabliau fort ancien dont des parallèles figurent dans les œuvres de La Fontaine, de Rabelais et d'Ésope.

«D'ordinaire, écrit Barbeau, c'est la jeunesse bachelière en mal de lettres et de sciences qui gravit la pente de la hautaine université. Cette fois - et ce n'est pas la seule depuis quelques années - c'était l'université même, par notre entremise, qui se penchait humblement sur un illettré de Cadie, qu'il serait faux d'appeler ignorant, puisque céans il nous en apprenait de son $\mathrm{cru}^{3}{ }^{3}$ »

Il poursuit : " Doit-on rester insensible à la valeur de tels dires, échantillonnés dans le fabliau de la hache perdue et de la sereine, qu'il convient d'appeler traditionnels et antiques? Ou encore, pouvez-vous mettre en doute la sincérité des folkloristes que nous sommes, dans leur poursuite du savoir à ses sources vives parmi le peuple ? Apprenez donc ici la détermination qu'ils ont prise, avec l'appui clairvoyant de leurs chefs, de l'incorporer désormais dans le programme universitaire des humanités : pas de licence ès lettres sans folklore ! ${ }^{4} »$

$$
* * *
$$

Cette anecdote ouvre l'article que Marius Barbeau livrait en 1954, dans un numéro canadien du Journal of American Folklore en 1954, sous le titre « Nos traditions à l'Université ». En plus d'illustrer l'originalité de la recherche ethnologique, qui savait s'alimenter à des sources orales dans un milieu intellectuel ne jurant encore que par l'imprimé, l'auteur annonce que l'Université Laval avait innové en intégrant la nouvelle science dans le cursus fondamental des humanités : «pas de licence ès lettres sans folklore ! , déclarait-il.

\section{Le programme}

Barbeau s'emploie alors à « énumérer à la hâte quelques thèmes et [à] indiquer certains champs d'études, qui nous sont bien connus et qui nous réclament d'urgence, note-t-il, mais que nous n'avons pas encore eu le temps ni l'occasion de divulguer. En descendant l'échelle du temps depuis l'antiquité jusqu'au soir récent à la Côte-au-canot », il brosse les grands champs de recherche de l'ethnologie d'alors.

2. Conte type Aa.-Th. 729 « La Hache tombée à l'eau »; Aarne-Thompson (« The Axe Falls into the Stream »), Aarne-Thompson-Uther (« The Merman's Golden Axe [La Hache d'or du triton »).

3. Marius Barbeau, « Nos traditions à l’Université », op. cit., p. 200.

4. Loc. cit. 
La première place revient aux contes populaires qui sont les plus anciens et « de même souche orale que les mythes classiques de la Grèce », tels la Bête-à-Sept-Têtes, le Phénix doré, dont la version Le Grand Sultan d'Édouard Hovington de Tadoussac; des contes à tiroir à la manière des contes arabes des Mille et une Nuits, comme Le Fin Voleur de Valenciennes, qu'Hérodote rapportait déjà et attribuait au roi Rhampsinite ; les parallèles des romans français de la Table ronde ou anglais du roi Arthur, et des conteurs comme Boccace. Tout juste après viennent les fabliaux, L'Eau de la fontaine de Paris, et les légendes de diable danseur ou bâtisseur d'église, de loups-garous, de revenants, de chasse galerie 5 .

Après les récits oraux, il passe ensuite aux chansons populaires, relevant des thèmes « issus du paganisme oriental et méditerranéen » (Dame Lombarde, Alexis), des miracles de l'ère chrétienne, des pays nordiques ( $L a$ Ignolée, le Roi Renaud), de la France historique (Les Écoliers de Pontoise), les chansons de métiers et à danser, à boire, de fêtes, d'amour, de l'enfance jusqu'aux chansons locales (La Complainte de Cadieux) et de voyageurs. Il s'attarde alors à l'art poétique et à la mélodie des chansons populaires ${ }^{6}$ : la métrique du vers, la césure épique et son corollaire, « la loi familière des césures inverses », la forme strophique, section qu'il conclut ainsi : " C'est un devoir urgent, à l'Université Laval, d'en entreprendre l'étude adéquate dans un avenir rapproché, d'autant plus que le répertoire immense de chansons populaires au Canada en fournit amplement la matière première, et que les premiers pas en cette direction ont déjà été franchis : l'auteur de ces lignes a donné plus d'une série de conférences sur cet art poétique ancien que le Canada, grâce à son isolement culturel, a su mieux conserver que la mèrepatrie. ${ }^{7} \gg$

Il achève cette partie de son exposé sur l'aspect musical de la chanson en survolant les modes anciens, « desquels est issu le plain chant», qui sont « plus typiques du répertoire que ne l'est le mode d'ut ou majeur et son relatif mineur ». Puis il cite Marguerite Béclard d'Harcourt qui connaît bien le répertoire d'ici et qui affirme que : «L'intérêt supérieur des versions canadiennes réside précisément en ce fait qu'elles nous permettent de compléter la physionomie du chant français en lui restituant quelques-uns de ses plus beaux traits. ${ }^{8} \gg$

Il considère enfin brièvement « les arts et métiers et le langage du peuple, qui tiennent aussi de la tradition populaire ${ }^{9} »$.

\footnotetext{
5. Marius Barbeau, op. cit., p. 203-204.

6. Ibid., p. 205-209.

7. Ibid., p. 209.

8. Loc. cit.

9. Marius Barbeau, op. cit., p. 209-210.
} 
En conclusion, il revient sur son « jeune pêcheur de Shippâgan » qui lui « rappelait le Bourgeois Gentilhomme, qui disait de la prose sans le savoir » et dont la prose, continue Barbeau, « était une parcelle du trésor incommensurable des traditions orales, trésor que, par l'apport de la langue, la vieille France a légué à ses fils d'Amérique. À l'Université maintenant de se pencher sur cette richesse inattendue! Si elle accomplit ce qu'elle doit - noblesse oblige - elle y retrouvera, sinon la gloire, du moins la joie de la découverte et de la création, par l'usage qu'elle en fera, l'indépendance spirituelle qui conduit à l'égalité et à la fraternité des nations unies. ${ }^{10}$ »

En appendice, figurent la transcription du conte La Sereine de mer et les trois haches et la fable d'Ésope Le Bûcheron et Hermès ${ }^{11}$.

\section{Le folklore selon Barbeau}

En considérant cet article, on s'imagine sans peine que la littérature orale était au cœur de l'enseignement qu'il professait à l'Université Laval avec la première équipe des Archives de folklore : contes, chansons, légendes, coutumes, jeux et divertissements, musique avec leur pendant littéraire qui leur servait à des fins documentaires pour la comparaison, sans oublier enfin la méthodologie de l'enquête orale qui fondait la spécificité de la discipline ${ }^{12}$.

En cette année du soixante-dixième anniversaire de la fondation des Archives de folklore (1944-2014), qui survient six décennies après sa parution, l'article de Marius Barbeau constitue un précieux repère pour apprécier le chemin parcouru, la manière dont l'Université Laval s'acquitte de sa tâche de présenter le «trésor incommensurable des traditions orales » dans son enseignement et le rang que celui-ci occupe dans les recherches qu' on y mène. Il permet aussi de jauger la place de la littérature orale dans les programmes contemporains des universités, ici et ailleurs.

10. Ibid., p. 210.

11. Ibid., p. 210-211.

12. Dans un ouvrage ultérieur, rappelant que « [s]elon leurs aptitudes et leurs goûts, [les premiers] folkloristes ont orienté leurs recherches vers des modes particuliers d'expression populaire » et que " [1]eurs tendances individuelles ont été de restreindre plutôt que d'amplifier le champ de leurs enquêtes sur le terrain, qui se fait trop vaste », il élargira le domaine et la définition du folklore : « Il reste encore beaucoup à entreprendre surtout dans la perspective des métiers et des arts populaires. » $C f$. Marius Barbeau, Folklore, « Cahiers de l’Académie canadienne-française » 9, Montréal, [1965], p. 8. 